Rev. salud pública. 14 sup (2): 100-112, 2012

\title{
Políticas en salud ambiental, con énfasis en contaminación atmosférica e infancia, en ciudades colombianas
}

\section{Environmental health policies emphasizing air pollution and childhood in Colombian cities}

\author{
Cesar A. García-Ubaque ${ }^{1}$, Juan C. García-Ubaque ${ }^{2}$ y Martha L. Vaca-Bohórquez ${ }^{3}$ \\ 1 Facultad de Tecnología, Universidad Distrital Francisco José de Caldas. Bogotá, Colombia. \\ cagarciau@udistrital.edu.co \\ 2 Facultad de Enfermería, Universidad Nacional de Colombia. Bogotá. Colombia. jcgarciau@unal.edu.co \\ 3 Universidad de Los Andes. Bogotá, Colombia.ml.vaca68@uniandes. edu.co
}

Recibido 25 Mayo 2011/Enviado para Modificación 8 Agosto 2011/Aceptado 3 Noviembre 2011

\section{RESUMEN}

Objetivo Se presentan los resultados del proyecto de investigación sobre salud ambiental en ciudades colombianas con énfasis en contaminación atmosférica, en respuesta a la iniciativa planteada en el Consejo Nacional de Política Económica y Social, CONPES 3344 de 2005.

Método El proyecto se desarrolló entre 2008 y 2009 para el Ministerio de Ambiente Vivienda y Desarrollo Territorial, bajo la metodología de Evaluación Ambiental Estratégica formulada en el Analytical Strategic Environmental Assesment Methodology ANSE, para lo cual se contó con la participación mesas de expertos en diferentes áreas.

Resultados Se identificaron cinco temas centrales o ejes temáticos sobre los cuales se debe fundamentar este componente de la política: 1 Planificación Urbana, 2 Movilidad y Transporte, 3 Industria y Servicios, 4 Fuentes de Energía y 5 Gestión del Riesgo; Dentro de cada eje temático se hizo un diagnóstico sobre su estado actual a partir del conocimiento de los expertos y de información oficial.

Conclusión Con base en los diagnósticos, se formuló un conjunto de acciones o intervenciones a ejecutar en diferentes niveles por parte de las entidades de estado involucradas. De manera complementaria se proponen recomendaciones para el proceso de toma de decisiones correspondientes a las intervenciones sugeridas. Finalmente se plantean los lineamientos metodológicos para la investigación sobre variables económicas y de vigilancia epidemiológica a tener en cuenta.

Palabras Clave: Salud ambiental, ciudad saludable, política ambiental municipal, planificación de ciudades, contaminación ambiental (fuente: DeCS, BIREME). 


\section{ABSTRACT}

Objective Responding to an initiative advanced by the National Economic and Social Policy Council (CONPES, 3344/2005), this article presents the results of a research project concerned with environmental health in Colombian cities emphasising air pollution.

Method The project was carried out between 2008 and 2009 for the Colombian Ministry of the Environment, Housing and Territorial Development using strategic environmental assessment (SEA) methodology contained in the complementary and decision-centred approach known as analytical strategic environmental assessment (ANSEA), relying on expert participation in different areas.

Results Five key issues were identified to base such policy on: urban planning, mobility and transport, industry and services, energy sources and risk management. Each theme was diagnosed regarding its current state from experts' knowledge and official information.

Conclusions Such diagnosis led to a series of actions or interventions to be implemented at different levels by the state entities involved. Complementary recommendations were made for decision-making relating to the suggested interventions. Methodological guidelines were proposed for research into economic variables and the pertinent epidemiological surveillance.

Key Words: Environmental health, urban health, policy, air pollution (source: MeSH, NLM).

$\mathrm{L}$

a Salud Ambiental es el área de las ciencias que trata los riesgos y los efectos que para la salud humana representa el medio en que ambiental considera también los cambios naturales o artificiales que el medio manifiesta, así como la contaminación producida por el hombre. Según la Organización Panamericana de la Salud OPS, la Salud Ambiental comprende los aspectos de la salud humana que están determinados por factores físicos, químicos, biológicos, sociales y psicosociales presentes en el ambiente (2). Este enfoque integral de la salud ha derivado en un nuevo enfoque de atención y prevención en salud que consiste en desarrollar ambientes saludables en los diferentes espacios en los que puede permanecer cualquier individuo a lo largo de su vida, como el espacio de vivienda ya sea temporal o permanente, el espacio ocupacional bien sea laboral o académico y el espacio público donde las personas desarrollan actividades comerciales, de recreación y de movilidad (3). En este enfoque se incluye la práctica y la teoría de evaluar, corregir, controlar y prevenir los factores y condiciones presentes en el ambiente que puedan afectar la salud de las diversas generaciones. 
Para el caso de las ciudades colombianas la contaminación atmosférica es uno de los factores que más incidencia tiene sobre la salud ambiental (4). Afecta principalmente a la población infantil y tiene un alto impacto económico por los costos asociados a la atención en salud y a los controles que demandan las fuentes generadoras de dicha contaminación. Este fenómeno involucra muchas variables y características que pueden llegar a tener diversos grados de complejidad que a su vez deben ser atendidas por diferentes entidades y organismos del estado tanto del orden nacional como local. Por esta razón se escogió la Evaluación Ambiental Estratégica EAE como el instrumento metodológico más adecuado para la formulación de los lineamientos de política en Salud Ambiental.

\section{METODOLOGIA}

La Evaluación Ambiental Estratégica (EAE) por definición es un proceso sistemático para evaluar consecuencias ambientales en propuestas de políticas, planes o programas y asegurar que en ellas se incluyan totalmente y hayan sido tratadas apropiadamente en las fases más tempranas factibles, los impactos ambientales, al mismo nivel que consideraciones de tipo social y económico (5). Así mismo, la EAE, se define como un instrumento de apoyo al proceso mismo de toma de decisiones de naturaleza estratégica, normalmente traducidas en políticas, planes y programas y se constituye como un proceso sistemático de identificación, análisis y evaluación previa de impactos (6). El proceso incluyó cinco pasos debidamente diferenciados así:

- Revisión y análisis por parte del equipo de trabajo de la documentación existente, focalizada en los ejes temáticos definidos por los términos fijados por el MAVDT

Estas acciones permitieron identificar los factores causales y posteriormente priorizar las acciones de acuerdo con el modelo de determinantes estructurales, intermedios y proximales siguiendo la metodología de la EAE. A partir de ellos, se desarrolló una etapa de análisis mediante un modelo sistémico de causalidad, postulado por Loyola (7), que integra los cinco ejes temáticos identificados: Planificación Urbana, Movilidad y Transporte, Industria y Servicios, Fuentes de Energía y Gestión del Riesgo, considerando además los aspectos de coordinación intersectorial.

- Entrevista con expertos nacionales y consulta grupal de expertos 
Se realizaron talleres de consulta a expertos en el contexto de cada eje temático estratégico definido, se construyeron diagramas o relaciones de causa-efecto para identificar nítidamente los factores que llevan a producir efectos nocivos sobre la salud ambiental y las políticas, programas y planes de mayor importancia en este sentido.

\section{- Talleres nacionales}

Para facilitar el desarrollo del proceso en colectivo, se empleó inicialmente el diagrama de relación causa-efecto conocido como diagrama de Ishikawa o diagrama de espina de pescado (8). Esta es una herramienta que ayuda a identificar, clasificar y poner de manifiesto posibles causas de problemas específicos (en este caso efectos nocivos sobre la salud ambiental). Ilustra gráficamente las relaciones existentes entre un resultado (salud ambiental) y los factores que influyen en el mismo. El ejercicio con el diagrama espina de pescado consistió en presentar por cada eje temático los principales factores que confluyen en la columna vertebral del diagrama señalando su importancia en provocar un impacto negativo en la salud ambiental. Estos diagramas se presentaron a los participantes para su evaluación crítica lo que implicó construir nuevos tipos de diagramas.

- Talleres regionales.

Como consecuencia de la discusión producida en el Primer Taller Nacional sobre los diagramas de relaciones causa-efecto, se decidió utilizar otro tipo de diagrama de la familia de relaciones causa-efecto, basado en el llamado modelo conceptual Fuerza Motriz-Presión-Estado-Impacto-Respuesta (9). El diagrama permitió organizar la información respecto al estado de la salud ambiental, a través de un ejercicio que utilizó el llamado Árbol del Problema. Este modelo plantea que las actividades del hombre impactan de alguna manera el ambiente y, cuando estos impactos son negativos, inducen a los humanos a restringir o controlar los factores que afectan alguna fase del sistema. Para este ejercicio, el modelo fue modificado en la forma que se muestra en la Figura 1.

Los factores estructurales son factores socio-económicos y políticos que causan o favorecen cambios en el medio ambiente, los cuales influencian negativamente los factores intermedios. Son factores en donde las decisiones se dan en los niveles más altos de formulación de políticas, planes y programas. 
Ejemplo de estos factores son las políticas que orientan la actuación de los diversos actores de la vida nacional, ya sean de la administración nacional o local, las industrias y aún las personas.

Los factores intermedios son factores antropogénicos que influencian directamente el estado del medio ambiente y cambian la calidad ambiental y la cantidad de los recursos naturales. Ejemplo de esto puede ser la actividad industrial y sus características, las manifestaciones de la planeación urbana en términos de ordenamiento de las actividades (ubicación de industrias, viviendas y aún factores como la pobreza y el desplazamiento).

Figura 1. Modelo conceptual para determinación de relaciones causa-efecto

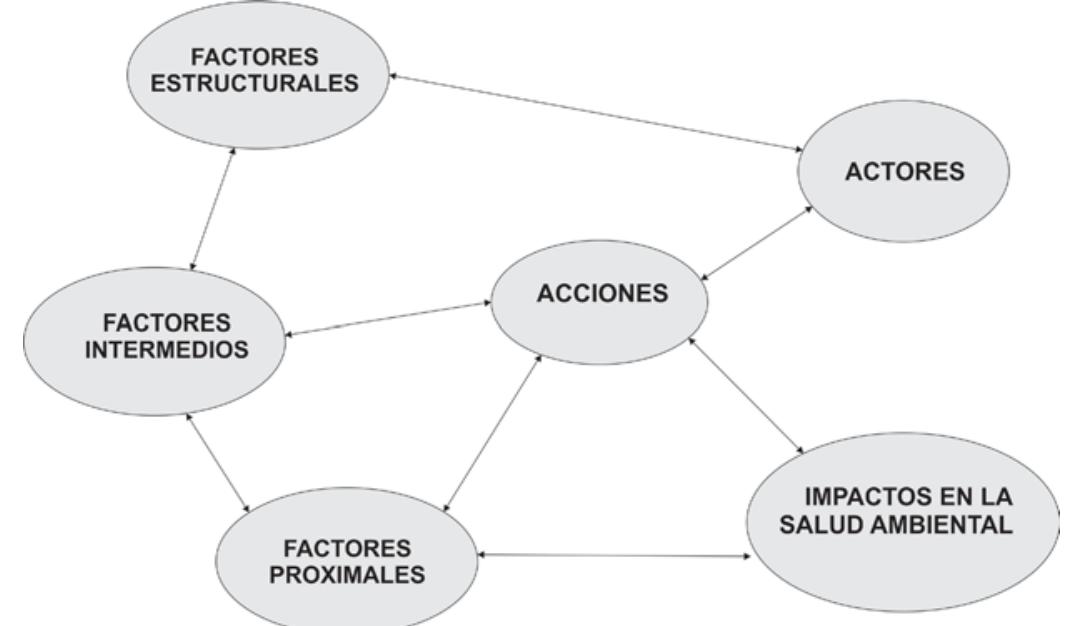

Modificado de: United States Environmental Protection Agency Secretaría de Medio Ambiente y Recursos Naturales de México (2006), Estrategia para el Desarrollo de Indicadores. Frontera 2012: Programa Ambiental MéxicoEstados Unidos, disponible en: http://www.zepa.gov/border2012/docs/bitf_dev_200608_esp.pdf

Los factores proximales hacen referencia a las exposiciones negativas a que son sometidas las personas debido a la actividad humana y sus consecuencias en el medio ambiente. Tales exposiciones se dan en la intersección entre las personas y los riesgos inherentes en el ambiente. Para este caso, se hace referencia directa a la exposición a gases, vapores y material particulado, así como a agentes químicos no convencionales. Para el caso del eje temático relativo a evaluación del riesgo, se dan una serie de exposiciones de carácter social que vinculan la relación de las personas con los servicios de salud y su calidad, así como a la vulnerabilidad diferencial que le ocasionan sus características de género, edad, genética, condición socioeconómica y estilo de vida. 
Los impactos son aquellos cambios producidos en la salud de las personas, referidos específicamente a enfermedades agudas y crónicas del aparato respiratorio, aunque son incluidas otras manifestaciones en la salud general de la población, en particular infantil.

Las acciones se referían en este caso a aquellas propuestas que deberían realizar los diversos actores relacionados con la problemática ambiental, en especial con los ejes temáticos.

Los actores son quienes intervienen en el proceso de toma de decisiones que buscan modificar las situaciones ocasionadas en cada nivel. Por ejemplo, para el nivel estructural, los actores están relacionados con quienes entran en el proceso de desarrollo de una política, el cual incluye la identificación del problema, la formulación de soluciones, la toma de decisión, la aplicación de la política como tal y la evaluación (10). Aun cuando la autoridad gubernamental (nacional, regional o local) es el actor central de una política pública, no es el único que interviene, ya que entra en una relación de interdependencia con otros actores: organismos encargados de ejecutar sus decisiones, sujetos que reaccionan a las realizaciones producidas, grupos de intereses u otras instituciones que ejercen influencia sobre la acción proyectada o en curso, trátese de una política, un plan o un programa.

Análisis y Elaboración del informe final

A partir de las discusiones, dadas en los talleres y el conocimiento y experiencia del grupo investigador en las temáticas tratadas, se desarrollaron los lineamientos de política para cada eje a partir de la elaboración de un árbol de problema para cada eje temático, ya que esta es una técnica participativa que ayuda a identificar el problema y a organizar la información recolectada, generando un modelo de relaciones causales que lo explican.

La lógica define que cada problema es consecuencia de los que aparecen debajo de él y, a su vez, es causante de los que están encima, reflejando la interrelación entre causas y efectos; para tal fin se procedió a realizar la Identificación del problema central: entendido como el impacto ocasionado sobre la salud ambiental por la contaminación atmosférica, la Identificación de factores proximales: aquellos básicamente comunes a todos los ejes y que hacen referencia a las exposiciones negativas a que son sometidas las personas debido a la actividad humana y sus consecuencias en el medio ambiente, estas 
se clasificaron en dos tipos: exposición por contaminación de de sitios abiertos (outdoor pollution) y exposición por contaminación de ambientes internos o cerrados (indoor pollution). A continuación se procedió a la Identificación de factores intermedios y estructurales buscando relacionar políticas, planes y programas existentes con los factores antropogénicos que influyen directamente el estado del medio ambiente y cambian la calidad ambiental y la cantidad de los recursos naturales, ocasionando problemas de salud ambiental y finalmente se llego a la Identificación de acciones y actores: que es necesario tener en cuenta para buscarle respuestas a la problemática ambiental ocasionada a partir de los componentes de cada eje temático.

A partir del modelo resultante se identificaron factores determinantes estructurales de carácter social, económico, político, ambiental, tecnológico y de biología humana, todos ellos relacionados entre sí y que a su vez tienen una estrecha interacción con el sistema de salud.

Como elementos de correlación, se establecieron factores determinantes intermedios que generan condiciones de vida deficientes, riesgos y peligros ambientales, así como, cambios en los estilos de vida y comportamiento, que alteran los niveles de esperanza de vida, inciden en la aparición de enfermedades, daños, discapacidades y muertes, afectando el bienestar de la población (11).

\section{RESULTADOS}

A partir del modelo planteado y del análisis detallado se encontraron las relaciones de causalidad, que permiten identificar los aspectos sensibles a tener en cuenta en el diseño de la política (Figuras 2, 3 y 4).

Se observa una fuerte relación entre los ejes temáticos de Planeación Urbana, Sector Industrial y de Servicios y Movilidad y Transporte. Aunque el eje temático Calidad de Combustibles tiene actualmente una estrecha relación con el de Movilidad y Transporte y también guarda relación con el de Sector Industrial y de Servicios, no presenta mayor relación con el de Planeación Urbana, de otra parte y por la importancia que tiene la vigilancia epidemiológica y la evaluación de riesgo en la población dentro del seguimiento de la problemática en salud ambiental por causa de la contaminación atmosférica en las ciudades colombianas, se consideró necesario incluir este eje temático de manera independiente (12). 
Figura 2. Modelo integrado de causalidad - Movilidad y transporte - Planeación Urbana - Sector Industrial y de Servicios

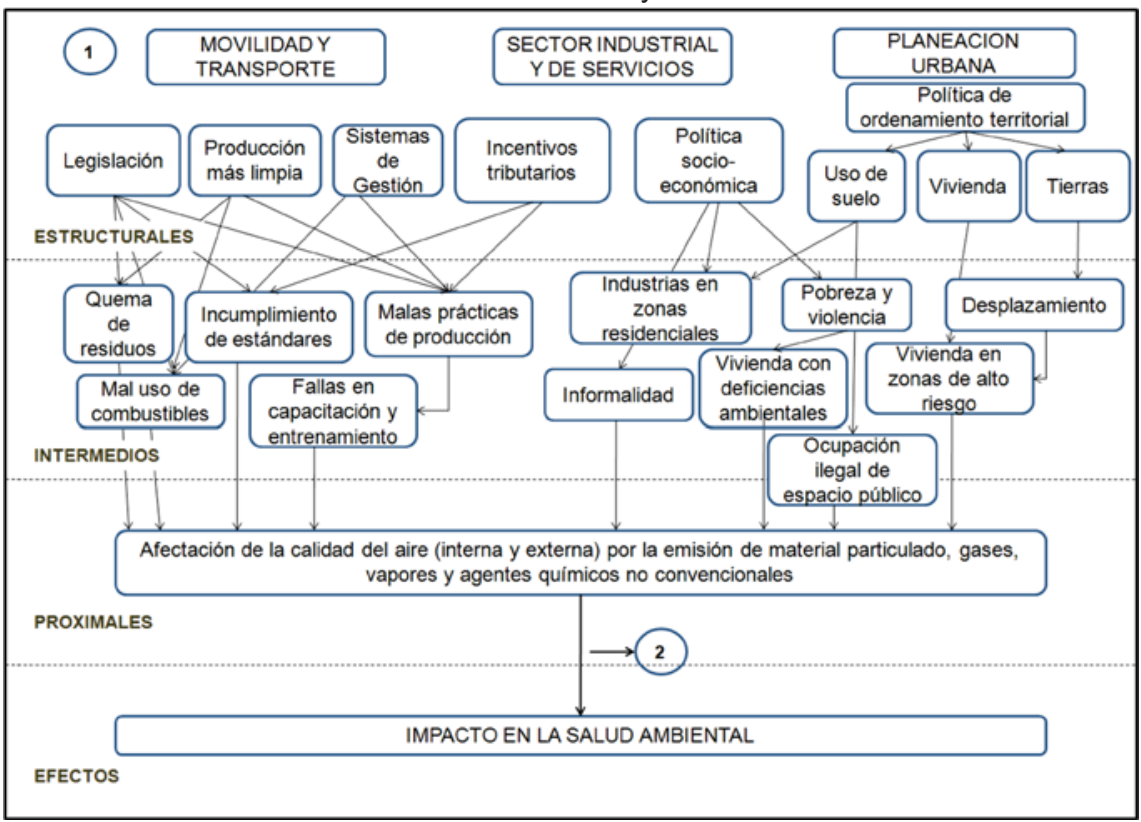

Figura 3. Modelo integrado de causalidad - Calidad de Combustibles

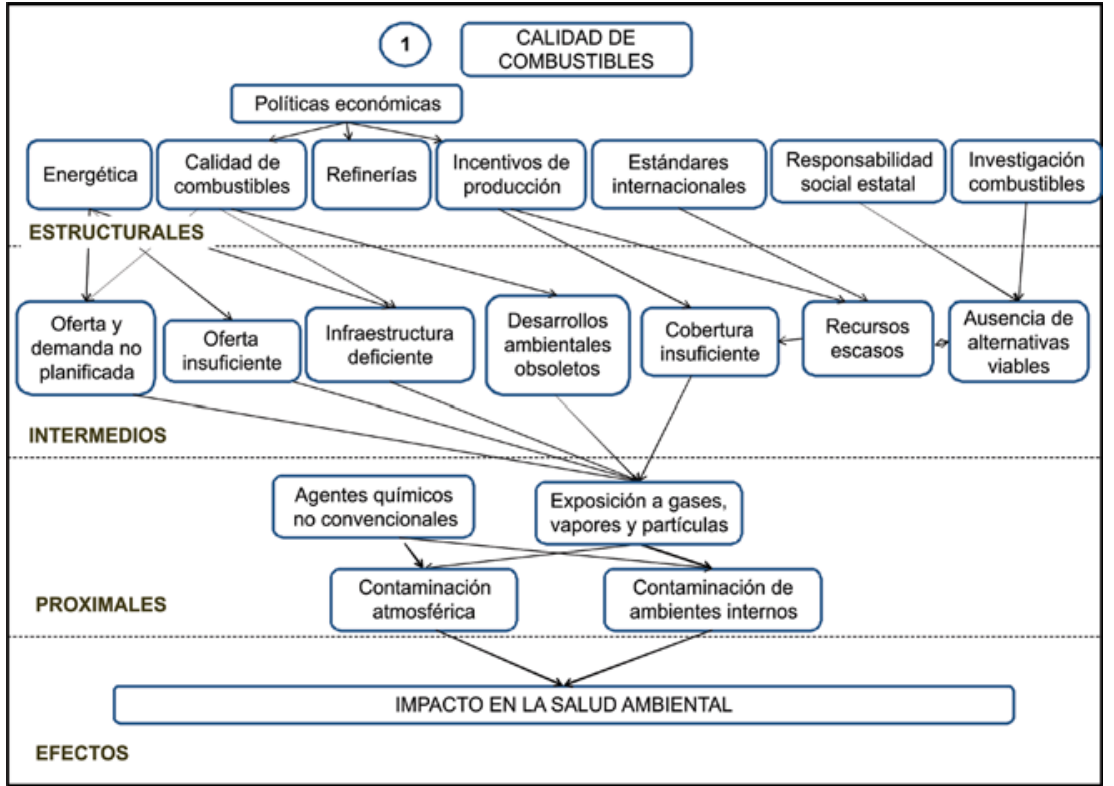


Figura 4. Modelo integrado de causalidad - Evaluación y Gestión del Riesgo

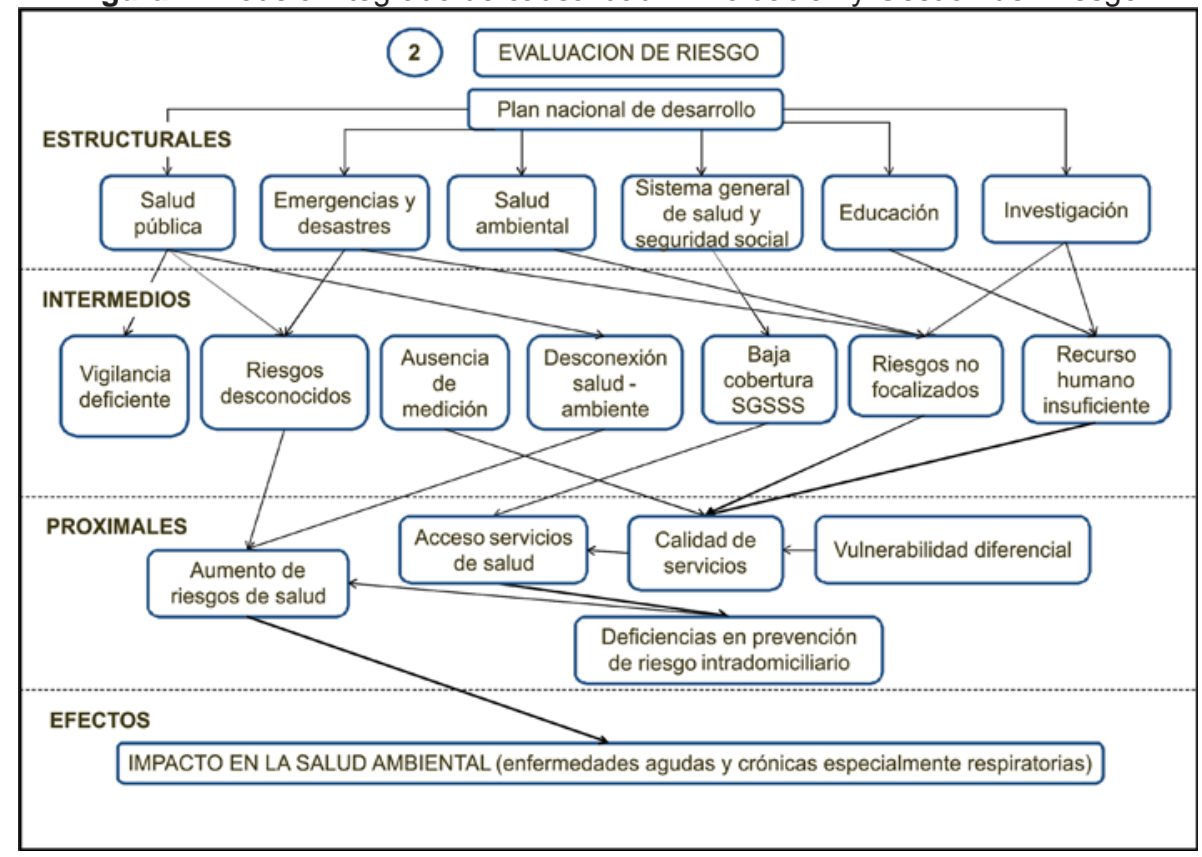

Con base en las relaciones de causalidad identificadas y de acuerdo con el contexto socioeconómico del país y su legislación vigente, se formularon las siguientes intervenciones para ser consideradas dentro de la política:

Planeación urbana, sector industrial y de servicios y movilidad y transporte

- Generar mecanismos de coordinación y de agendas interinstitucionales entre las entidades gubernamentales encargadas de la salud, el medio ambiente y el desarrollo económico, que permitan promover líneas de acción que integren propósitos nacionales, regionales y locales.

- Desarrollar actividades de vigilancia y control de los Planes de Ordenamiento Territorial de acuerdo con variables de salud ambiental.

- Fortalecimiento de la "gerencia local eficiente" a nivel municipal, generando mecanismos intersectoriales y promoción de lainterconexión o articulación de capitales en el entorno regional. 
- Fortalecimiento de mecanismos sancionatorios para el incumplimiento de la normatividad ambiental.

- Definición de criterios y estrategias para la política urbana, que incluyan: reubicación de zonas industriales incompatibles y planificación del uso del suelo.

- Fortalecimiento de procesos de investigación en el área de producción más limpia mediante cofinanciación de proyectos.

- Promoción de incentivos a empresas que establezcan programas de producción más limpia.

- Renovación del parque automotor y estimulación de sistemas de transporte masivo.

Calidad de fuentes energéticas

- Realizar planeación energética a nivel nacional, mediante la construcción de inventarios de oferta y demanda e introducción de combustibles más limpios que tengan en cuenta el balance de emisiones en toda la cadena de producción: transporte, manejo, distribución y uso final y promover que su cobertura sea de carácter nacional, evitando desigualdades.

- Promover incentivos para la utilización de energías renovables: eólica, solar, etc., de acuerdo con condiciones regionales.

- Promover la investigación para el desarrollo de energía proveniente de fuentes diferentes al combustible fósil.

- Evaluar el balance costo-efectivo de los biocombustibles y su impacto en la salud ambiental.

Evaluación y gestión de riesgo

- Construcción del "Sistema Único de Vigilancia" como herramienta de planeación nacional, regional y local.

- Fortalecimiento de líneas de investigación en procesos relacionados con la sinergia salud-ambiente, identificando: factores deteriorantes y protectores y nuevas formas de intervención.

- Implementación de Planes Integrales de Entornos Saludables en las ciudades. 
- Elaboración de un manual actualizado de prevención de riesgos de ejecución obligatoria en todas las entidades relacionadas con atención en salud.

- Cualificación y fortalecimiento del talento humano requerido para llevar a cabo el proceso, en las etapas de construcción, implementación, seguimiento y evaluación.

- Promover la cátedra de "salud ambiental" dentro de la carrera de Medicina y carreras paramédicas y la cátedra de "prevención de la contaminación" en todas las instituciones educativas.

- Establecer métodos de evaluación y seguimiento de las estrategias de vigilancia epidemiológica en el país.

\section{DISCUSIÓN}

En los diferentes estudios ambientales hechos en Colombia se observa que no existe una interrelación seria con otros estudios sobre los efectos en la salud. De manera similar, los estudios de salud dejan de lado la parte ambiental dentro de sus resultados; lo cual, no permite una articulación adecuada de la información científica que, para el caso de la contaminación ambiental, es clave en los procesos de toma de decisiones. Esto podría superarse si las instituciones públicas como el Ministerio de Ambiente, Vivienda y Desarrollo Territorial y el Ministerio de Protección Social y otras, propenden por la interconexión de esta información en los procesos de toma de decisiones. Debería existir un observatorio de calidad de aire y salud para que trabajen conjuntamente con las oficinas de salud locales y los Ministerios de Ambiente y Salud con el fin de mejorar la capacidad de información científica de las entidades por medio de programas de innovación tecnológica y, por ende, debe existir un compromiso del sector industrial respecto al tema de salud ambiental.

La investigación encontró que para las ciudades colombianas el principal eje temático a considerar en cuanto a política de salud ambiental es la Planeación Urbana. Se puede afirmar también, que este eje temático debe ser el centro de las acciones a tomar ya que es articulador de los demás ejes temáticos considerados (12).

Las siguientes acciones son las recomendaciones derivadas del proyecto y que a juicio del grupo de investigación deben ser tenidas en cuenta de 
manera prioritaria con el fin de atender la problemática de la contaminación atmosférica en las ciudades colombianas:

- Coordinación inter e intrasectorial y articulación de políticas.

- Fortalecimiento de políticas sociales

- Planeación urbana sostenible.

- Construcción e implementación técnica, administrativa y financiera continuada que fortalezca el abordaje de los diferentes determinantes que afectan las condiciones de calidad de vida y salud.

- Establecimiento de un sistema de vigilancia y control de emisiones atmosféricas en fuentes fijas y móviles.

- Determinación del perfil de morbimortalidad nacional y regional relacionado con la contaminación atmosférica.

- Investigación en salud ambiental y formación de talento humano en el tema.

- Fortalecimiento de procesos de cultura y participación ciudadana.

- Acceso a servicios de salud.

- Construcción de vías de tránsito y fomento del uso de transporte masivo y otros medios no motorizados

\section{REFERENCIAS}

1. Ordóñez G. Salud Ambiental: conceptos y actividades. Revista Panamericana de la Salud Pública. 2000; 7(3):137-147.

2. PNUMA-OPS. [Internet]. Evaluación de las metodologías de medio ambien2. te y salud en América Latina y el Caribe; 2005. Disponible en: http://aplicaciones.medioambiente. gov.ar/ archivos/web/saludambiente/File/Evaluacion.pdf. Consultado 6 de mayo de 2008.

3. García-Ubaque JC. Hábitos Saludables en trabajadores hospitalarios. Tesis Doctoral Doctorado en Salud Pública Universidad Nacional de Colombia; 2011.

4. Onursal B, Gautam S. Contaminación atmosférica por vehículos automotores, experiencias recogidas en siete centros urbanos de América Latina. Banco Mundial Documento Técnico № 373S, 1997.

5. Colombia, Ministerio de Ambiente, Vivienda y Desarrollo Territorial MAVDT. Lineamientos de política de energéticos incluidos los combustibles líquidos y sus precios en Colombia; 2006.

6. Partidário MR. Conceptos, evolución y perspectivas de la Evaluación Ambiental Estratégica. Seminario de Expertos sobre la Evaluación Ambiental Estratégica en Latinoamérica en formulación y gestión de políticas. Santiago de Chil; 20 al 22 de noviembre de 2006.

7. Loyola E. Progress on Children's Environmental Health in the Americas, International Conference for the Evaluation of Global Health Strategies, Florence, Italy, 2006. Citado en: OPS (2007), Salud en las Américas 2007. Washington, p. 223.

8. Diagrama espina de pescado [Internet]. Disponible en: http://www.virtual.unal.edu.co/ cursos/sedes/manizales/4100002/lecciones/instrumentos/causaefecto.htm Consultado el 30 de enero 2010. 
9. United States Environmental Protection Agency-EPA-Secretaría de Medio Ambiente y Recursos Naturales de México. Estrategia para el Desarrollo de Indicadores. Frontera 2012: Programa ambiental México-Estados Unidos; 2006. [Internet]. Disponible en: <http://www. epa .gov/border2012/docs/bitf_dev_200608_esp.pdf>.

10. Meny I, Thoenig JC. Las políticas públicas, Barcelona, Editorial Ariel, S.A; 1992.

11. Cohen E, Martínez R. Manual de Formulación, evaluación y monitoreo de proyectos sociales, CEPAL; 1996.

12. Colombia, Ministerio de Ambiente, Vivienda y Desarrollo Territorial MAVDT. Evaluación Ambiental Estratégica (EAE) para la formulación de política en materia ambiental para Colombia, con énfasis en contaminación atmosférica en centros urbanos. Documento Técnico ASS/1487-08. Bogotá; 2008. 